\title{
Computed Tomography of the Pelvis without Contrast
}

National Cancer Institute

\section{Source}

National Cancer Institute. Computed Tomography of the Pelvis without Contrast. NCI

Thesaurus. Code C137904.

Computed tomography of the pelvis without the use of a contrast agent. 